\title{
Optimization of MSCT Tracking Images on Ureters against Noise Assessment with ASIR Variations
}

\author{
Nanang Sulaksono ${ }^{1, *}$, Jeffri Ardiyanto ${ }^{1}$, Vederica Farida Candra $^{1}$ \\ ${ }^{1}$ Poltekkes Kemenkes Semarang- Indonesia
}

\begin{abstract}
MSCT is one of the modalities to diagnose kidney function. Urinary tract examination or by using tracking reconstruction without using positive contrast media is able to produce urinary tract images and use tracking able to identify urinary tract images with urolithiasis. The need for an appropriate ASIR setting value in the MSCT plane parameters, To prove differences in image quality and identify which ASIR is best for MSCT too knowing the optimal image between using tracking and CT Urography examination using contrast. This experimental study with the PostTest Only Group Design study design, the number of samples used was 5 groups. The results of MSCT image showed that there was a significant difference in the value of $<0,001(\mathrm{p}<0.05)$ with the mean value for each ASIR as follows 20 mean value 3.40 , ASIR 40 mean value 7.90, ASIR 60 mean value 12.70, ASIR 80 mean value 18.00 , ASIR 100 mean value 23.00. The results of the MSCT urinary tract analysis after the ASIR test showed that there were significant differences between ASIR. Conclusion optimal image results using tracking by The mean value is ASIR 100 the rank of 23.00 and CT Urography using positive contrast media of 8.00 .
\end{abstract}

Keywords: MSCT; Ureter; Tracking; ASIR.

\section{Preliminary}

Urinary tract (urinary system) consists of two kidneys and ureters, urinary vesicles, and ureters. The kidneys are organs of excretion in the abdominal cavity, shaped like machines and are one part of the urinary system [1]. In Indonesia, there were 16,040 people, Central Java dominated by the second most in Indonesia after West Java [2].

Several ways to check the abnormalities of the urinary tract radiologically. These include plain abdominal photographs, intravenous pyelography, retrograde pyelography, antegradation of pyelography, cystography, ultrasonography, computed tomography (CT-Scan), and nuclear magnetic resonance (NMR) / magnetic resonance imaging (MRI). Excess CT scanning modalities can produce volumetric images (the ability to make thin pieces in a spiral) so that they can detect the intraabdominal organs in general and the urinary tract [3].

Urinary tract or CT Urography examination is generally carried out using positive contrast media, whereas according to [4], using tracking reconstruction in abdominal MSCT without using positive contrast media is able to produce urinary tract images and use tracking capable of identifying urinary tract images with urolithiasis cases in the form of stones in the ureter with contrast analysis [5].

On the MSCT tracking examination on the urinary tract, there are several parameters used, including slice thickness, pitch, FOV, kV, mAs, window width, window level, and ASIR. Image quality on MSCT is assessed from several components and each is influenced by several technical parameters. Components that affect the image quality on MSCT include: 1). Spatial resolution, 2). Contrast resolution, 3). Image noise, 4). Artifacts. ASIR is one parameter of image quality on MSCT. ASIR can influence contrast resolution, spatial resolution and then reduce the presence of noise [6].

In Indonesia, the use of tracking in the urinary tract is still not done, because many radiographers do not yet know the tracking software available on MSCT. In order to make the diagnosis more accurate, it is necessary to improve the quality of tracking images on the urinary tract with the proper use of ASIR, so that the images obtained are without noise. Optimal image results using tracking with the right ASIR will be compared with CT Urography examination using contrast.

\section{Method}

The design of this study is experimental with Posttest Only Group Design. The research subjects were 5 patients from each group, carried out variations of ASIR 20. 40. $60,80,100$ so that the number of images obtained was 25 images. The tracking image results using ASIR are optimal compared to CT Urography images using contrast media, to find out which image is optimal in providing diagnostic information on the urinary tract.

This study was conducted by evaluating abdominal MSCT image information, assessment of anatomical structure of image information on abdominal MSCT examination without positive contrast media [7].

\footnotetext{
*Corresponding author: nanang.sulaksono@yahoo.com
} 
Evaluation of image quality is done through a subjective method known as VGA, to assess whether the image matches what has not been determined by doing a relative assessment, where the value is given to the image quality, giving a value of 1-5 on MSCT criteria Urography, system classification used in absolute visual analysis of grading (VGA) - Study [8]

Assessment of MSCT Urography after the use of ASIR variations was carried out by 3 radiologists as observer, to test the suitability of the opinions of the three radiologists conducted by the Kappa test. If results are greater than $75 \%(\mathrm{k}>0.75)$, then the kappa test is said to be perfect.

Bivariate analysis was performed to test the differences in MSCT image of the urinary tract after the use of filter variations. Before the bivariate test, the data normality test was carried out using the Shapiro Wilk test $(\mathrm{n}<50)$. If the data is normally distributed $(\mathrm{p}>0.05)$, the One Way Anova test is followed by the Tukey Post Hoc test, and if the data is abnormally distributed $(p<0.05)$ the Kruskal Wallis test is then followed by the Mann Whitney test.

\section{Results}

\subsection{The results of the analysis of the urinary tract image are optimal for the use of tracking with ASIR variations.}
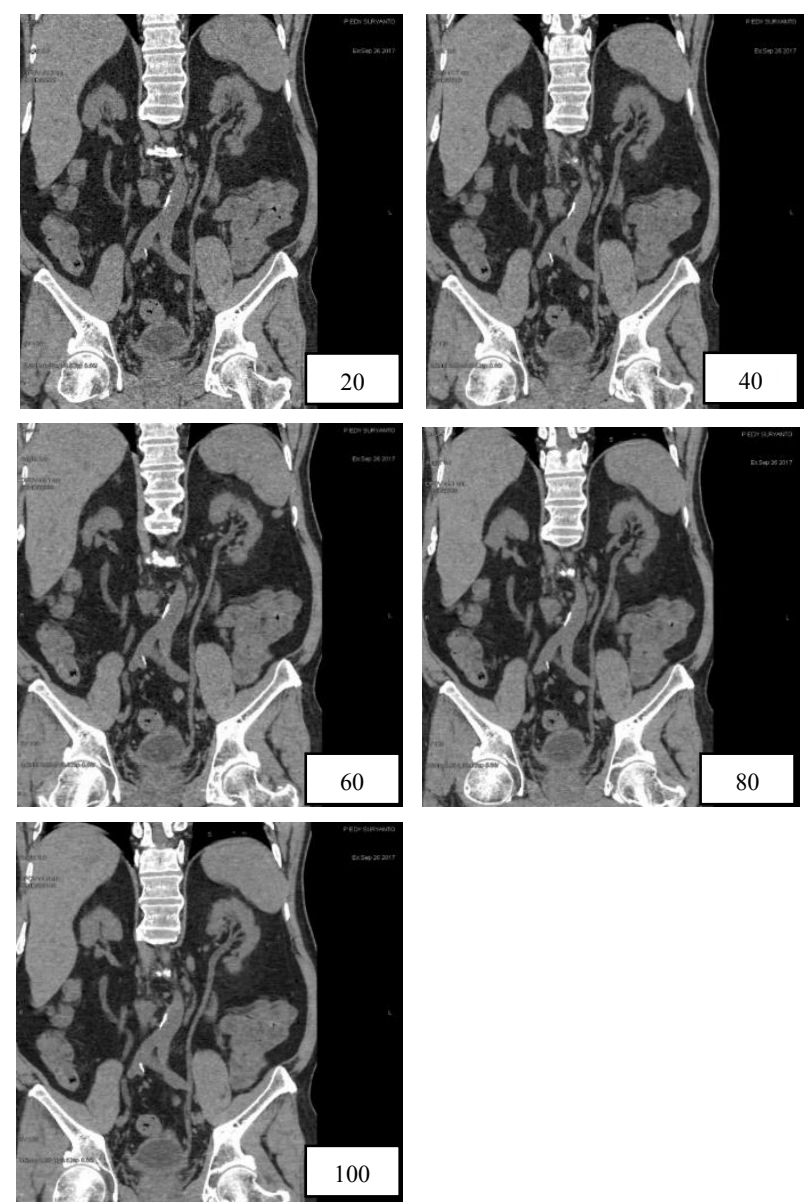

Fig 1. Image of urinary tract using tracking with ASIR 20, 40, $60,80,100$
The study was carried out after getting a research mark from the Health Research Ethics Commission of the Health Ministry of Health Semarang.

To request the conformity of the opinions of the three radiologists, a suitability analysis was carried out between observers using the Kappa test. The results of the Kappa test for the image review obtained perfect results $(k>$ $0.75)$, namely $\mathrm{k}=0.820$.

Patients who underwent abdominal MSCT examination without using positive contrast media included 25 inclusion and exclusion criteria. Optimal analysis of urinary tract images on tracking use with variations of ASIR 20,40.60,80,100, Normality test results are shown in Table 1.

Table 1. Test results of Normality with Shapiro Wilk Test

\begin{tabular}{|c|c|c|}
\hline Variable & $\mathrm{P}$ & Keterangan \\
\hline ASIR Variation & 0,000 & Not Normal \\
\hline
\end{tabular}

The results of the normality test using Shapiro Wilk, from variations of ASIR, obtained abnormal distribution data $(p<0.05)$, so that the Kruskal Wallis test was conducted, then continued using Mann Whitney analysis.

Image analysis of MSCT urinary tract between each group after tracking with a variety of filters. The Kruskal Wallis test results are shown in Table 2.

Table 2. Results of the Kruskal Wallis MSCT urinary tract test with variations of ASIR

\begin{tabular}{|l|c|c|}
\hline \multicolumn{1}{|c|}{ Variable } & Mean & P \\
\hline ASIR 20 & 3.4 & \\
\cline { 1 - 2 } ASIR 40 & 7.9 & \multirow{2}{*}{0,000} \\
\cline { 1 - 2 } ASIR 60 & 12.7 & \\
\cline { 1 - 2 } ASIR 80 & 18 & \\
\hline ASIR 100 & 23 & \\
\hline
\end{tabular}

Based on Table 2, it can be seen that the results of the MSCT image analysis of the urinary tract using tracking with ASIR variations showed there were significant differences with the p-value of $0.000(p<0.05)$, so that the Mann Whitney analysis continued. The results of the Mann Whitney test using tracking with ASIR variations are shown in Table 3.

Table 3. Results of the Mann Whitney test with variations of ASIR.

\begin{tabular}{|l|c|c|c|c|}
\hline Variabel & 40 & 60 & 80 & 100 \\
\hline 20 & 0,015 & 0,008 & 0,007 & 0,000 \\
\hline 40 & & 0,014 & 0,006 & 0,004 \\
\hline 60 & & & 0,007 & 0,006 \\
\hline 80 & & & & 0,006 \\
\hline
\end{tabular}


Based on Table 3, it can be seen the quality of MSCT images of the urinary tract after using tracking with ASIR variations, indicating that there are significant or significant differences between each ASIR group.

\subsection{Results Analysis of urinary tract images using ASIR 100 tracking with CT Urography using positive contrast media.}
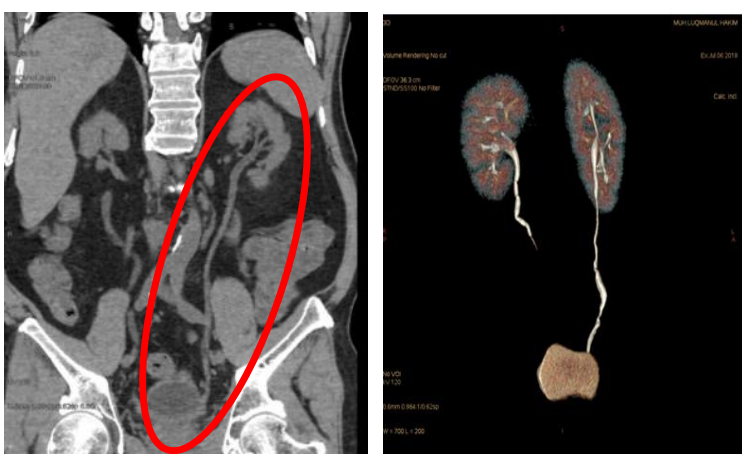

Fig 2. Image of the urinary tract, (a) using the ASIR 100 tracking, b CT Urography using positive contrast media

Analysis of the MSCT image of the urinary tract between each group after using ASIR 100 tracking with CT Urography using positive contrast media. The Kruskal Wallis test results are shown in Table 4.

Table 4. The results of the Kruskal Wallis MSCT test of the urinary tract using ASIR 100 tracking with CT Urography using positive contrast media.

\begin{tabular}{|l|c|c|}
\hline Variabel & Mean & $\mathrm{P}$ \\
\hline Tracking & 3 & 0,007 \\
\hline Media kontras positif & 8 & \\
\hline
\end{tabular}

Based on Table 4, it can be seen that the results of MSCT image analysis of the urinary tract using ASIR 100 tracking with CT Urography using positive contrast media showed that there were significant differences with a p.value of $0.007(p<0.05)$, followed by Mann Whitney analysis. The Mann Whitney test results are shown in Table 5.

Table 5. The results of the Mann Whitney test using ASIR 100 tracking with CT Urography using positive contrast media.

\begin{tabular}{|c|c|c|c|c|}
\hline Variabel & $\begin{array}{c}\text { Mann- } \\
\text { Whitney U }\end{array}$ & $\begin{array}{c}\text { Wilcoxo } \\
\mathrm{n} \mathrm{W}\end{array}$ & $\mathrm{Z}$ & Sig. \\
\hline $\begin{array}{c}\text { Tracking } \\
\text { CT } \\
\text { Urography }\end{array}$ & 0,000 & 15.000 & -2.694 & 0,008 \\
\hline
\end{tabular}

Based on Table 5, it can be seen the quality of MSCT images of the urinary tract using ASIR 100 tracking with CT Urography using positive contrast media, indicating that there are significant or significant differences.

\section{Discussion}

Analysis of the urinary tract MSCT image after ASIR variation with the Mann Whitney test showed that there were significant differences between the ASIR 20 group and ASIR 100 (p.value 0,000), ASIR 40 with ASIR 100 (p.value 0.004), ASIR 20 with ASIR 60 (p.value 0.008 ), ASIR 20 with ASIR 80 (p.value 0.007), ASIR 40 with ASIR 80 (p.value 0.014), ASIR 40 with ASIR 80 (p.value 0.006), ASIR 60 with ASIR 80 (p.value 0.007), ASIR 60 with ASIR 100 (p-value 0.006), ASIR 80 with ASIR 100 (p.value 0.006).

On the results of 25 urinary tract images using tracking with ASIR variation between each group, that the results of MSCT image analysis of the urinary tract between each ASIR group 20, 40, 60, 80, 100 showed a significant or significant difference with the $p$-value $0,000(p<0.05)$, meaning that Ho is rejected or Ha is accepted. This shows that there are significant differences in ASIR variation on image quality with a mean rank ASIR 20 of 3.40 , ASIR 40 of 7.90 , ASIR 60 of 12.70 , ASIR 80 of 18.00 , ASIR of 100 of 23.00 . So as to get the best ASIR with the lowest noise on ASIR 100 images and get an optimal image on the urinary tract.

On the results of the image using ASIR 100 tracking with CT Urography using 3D positive contrast media, differences were found, indicating differences with a p.value of $0.008(p<0.05)$. This shows that there are differences with the mean value of ASIR 100 tracking rank of 3.00 and CT Urography using positive contrast media of 8.00. so that the optimal anatomical assessment of MSCT in the urinary tract on examination using positive contrast media was obtained.

In tracking the use ASIR 100 can display and assess anatomy in renal parenchymal, renal and parenchymal appearance, proximal ureter, perirenal space, bladder, but for clarity of anatomical image the perirenal tracking space cannot show clearly.

\section{References}

1. Sanders, Tina, and Valerie CS, Textbook of Anatomy \& Physiology, ed, Medical Book Publisher EGC. Jakarta. (2007)

2. Pernefri, Dialysis Consensus, Issue in Publisher: Indonesian Nephrology Association FK UI: Jakarta. (2012)

3. A. Kawashima, T. Vrtiska, A. LeRoy, R. Hartman, MC. Collough, B. King, CT Urography. Radiological Society of NorthAmerica(RSNA) (2004)

4. N. Sulaksono, Suryono, J. Ardiyanto, Optimization of MSCT Image Urinary Tract Using Tracking with Slice Thickness Variation and Settings Window, Jurnal Riset Kesehatan. 5, 1: 30-34 (2016) 
5. N. Sulaksono, Suryono, J. Ardiyanto, The Optimalization of Uretherolithiasis Image with a Contrast Analysis on MSCT of Urinary Tract with Variation of Slice Thickness and Window Setting, American Scientific Publishers. 23, 2277-2280 (2017)

6. Amarudin, Image Quality (2007)

7. European Commission, European guidelines on quality criteria for computed tomography, Luxembourg. EUR 16262 (2000)

8. E. Ludewig, A. Richter, and M. Frame, Diagnostic Imaging Evaluating Image Quality Using Visual Grading Characteristic (VGA) Analysis, Springer Science Busines Media B.V (2010)

9. T. Niemann, V. Straten, C. Resinger, T. Bayer, G. Bongartz, Detection of Urolithiasis Using Low-Dose Simulation Study, Uropean Journal of Radiologi (2010)

10. A. O’Connor, Pathology, Mosby (2007) 